\title{
Wiederauffindung der Planeten (206) Hersilia und (210) Isabella.
}

Ich habe bereits Dr. Stechert von dem glücklichen Erfolge in Bezug auf Hersilia benachrichtigt. Heute theile ich eine zweite Beobachtung mit, welche Dr. S. Oppenheim angestellt hat und die bestätigt, dass der gefundene Planet dieselbe tägliche Bewegung hat wie der gesuchte, demnach also Hersilia ist. Er erhielt:

Dec. $168^{\mathrm{h}} 10^{\mathrm{m}} 8^{\mathrm{s}}$ M. Z. Wien

Stern wie Dec. $144^{\mathrm{h}} 9^{\mathrm{m}} 11^{\mathrm{s}} \cdot 25+15^{\circ} 6^{\prime} 33^{\prime \prime} \cdot 9$

\begin{tabular}{|c|c|c|c|c|}
\hline & & & $\begin{array}{r}4.83 \\
0.54\end{array}$ & $\begin{array}{r}+3.7 \\
-231.4\end{array}$ \\
\hline Planet & 4 & 4 & $\begin{array}{l}15 \cdot 54 \\
9 \cdot 346_{n}\end{array}$ & $\begin{array}{r}+154 \quad 6.2 \\
0.705\end{array}$ \\
\hline
\end{tabular}

Währing 1884 Dec. 17 .
Nachdem ich Hersilia erledigt hatte, machte ich mich an die Aufsuchung der Isabella auf Grund der umfassenden Rechnungen des Hrn. Wolyncewicz*) in Pulkowa. Ich war so glücklich in Folge des Umstandes, dass mir eine gute Sternkarte für diese Gegend zur Verfügung stand, den Planeten sehr rasch ohne Mühe zu finden.

Derselbe ist $\mathbf{I}_{2} \mathrm{~m}_{2}$ und wurde beobachtet:

Dec. $16 \quad 10^{\mathrm{h}} 0^{\mathrm{m}} 3^{\mathrm{s}}$ M. Z. Wien

Stern BB. VI $16257^{\mathrm{h}} 4^{6^{\mathrm{m}}} 13^{\mathrm{s}} 43+29^{\circ} 7^{\mathrm{\prime}} 35^{\prime \prime} 7$

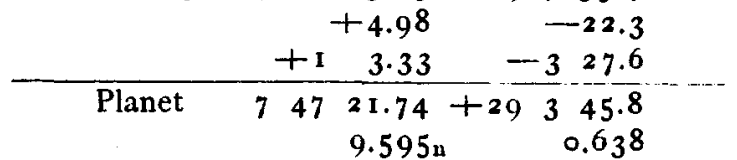

'Tägl. Bewegung laut Ephemeride: $-50^{\text {s }}$ und +4 '.

F. Palisa.

*) Ich hatte die Aufsuchungsephemeriden, die Hr. Wolyncewicz für die A. N. eingesandt hatte, nicht abgedruckt, sondern nach Wien geschickt, weil sie vielen Raum beanspruchten und weil nicht anzunehmen war, dass ausser Hrn. Dr. Palisa sonst Jemand sich mit der Aufsuchung befassen würde.

\section{Beobachtungen des Cometen 1884 Wolf.}

Auf der Herzogl. Sternwarte zu Gotha.

\begin{tabular}{|c|c|c|c|c|c|c|c|c|c|c|c|c|c|}
\hline 1884 & M.Z.Gotha & $\Delta \alpha$ & & $\Delta \delta$ & Vergl. & $\alpha \mathrm{al}_{1}$ & pp. & $\log p .4$ & $\delta \mathrm{a}_{1}$ & ipp. & $\log p .4$ & Red.ad I. app. & * \\
\hline pt. 25 & $1 \mathrm{I}^{\mathrm{h}} 23^{\mathrm{m}} 20^{\mathrm{s}}$ & $-0^{m} 5^{2.01}$ & + & $7^{\prime} 46 " 7$ & 5 & $21^{\mathrm{h}} 18^{\mathrm{n}}$ & $0.4 c$ & .354 & $+20^{\circ}$ & $8^{\prime} 54^{\prime \prime} 4$ & .669 & $+3^{5} \cdot 37+30.11$ & \\
\hline 25 & $12 \bigcirc 43$ & -3.19 .74 & - & $5 \quad 11.4$ & 5 & $21 \quad 18$ & I.95 & & +20 & & 687 & $+3.39+3$ & \\
\hline 26 & $10 \quad 1334$ & -0 I 4 & + & $7 \quad 4.6$ & 6 & 2118 & 37.7 & 109 & +194 & I $\quad 55.8$ & $65^{x}$ & $.3^{6}+30.1$ & \\
\hline 27 & $1 \mathrm{I} \quad 45^{\circ}$ & +235. & - & 1.4 & 12 & I 19 & & & +191 & 30 & 674 & $34+30.2$ & \\
\hline 29 & $\times 3 \quad 18 \quad 53$ & 一I 51. & -3 & 353.2 & 14 & 2 I 2 I & 06 & & +181 & $\begin{array}{ll}322.8 \\
\end{array}$ & 749 & $34+30.1$ & \\
\hline ct. 15 & $\begin{array}{lll}7 & 18 & 25\end{array}$ & +125. & - & $237 \cdot 3$ & 12 & 2139 & & & +103 & 26.3 & & $22+29.3$ & \\
\hline 15 & I0 4933 & +252.09 & + & 933.0 & $3 \cdot 5$ & 2 I 39 & & & +102 & $7+9.9$ & $75^{8}$ & $+3.2 x+29.3$ & \\
\hline It & 91428 & 一 34. & -1 & 246.4 & 10 & $2 \mathrm{I} 4 \mathrm{I}$ & & & +10 & 14.0 & & $2 \mathrm{I}+29.3$ & \\
\hline 16 & 10 555 & -I 10 & - & 730 & 5 & $214 \mathrm{I}$ & & & +95 & 959.1 & & $3+$ & \\
\hline 18 & $57 \quad 52$ & +314 & +1 & 238.9 & I 3 & 2 I 44 & & & +9 & $\begin{array}{ll}3 & 27.3\end{array}$ & & $20+28.9$ & \\
\hline 23 & $\begin{array}{lll}7 & 3 & 4\end{array}$ & -217 & - & 447.0 & I 6 & 2 I 52 & $5^{6}$ & $8.79 I_{n}$ & +64 & $9 \cdot 3.2$ & & $.21+28.4$ & \\
\hline 25 & $104 I 46$ & $+43^{6.83}$ & + & 534.1 & 9 & 2 I 56 & 53.90 & $9 \cdot 41 \mathrm{I}$ & +55 & $2 \quad 26.3$ & 0.788 & $+3.16+28.1$ & \\
\hline
\end{tabular}

Mittlere Oerter der Vergleichsterne für $\mathbf{r} 884.0$

\begin{tabular}{|c|c|c|c|}
\hline * & $\alpha \times 884.0$ & $\delta 1884.0$ & Autorität \\
\hline $\mathbf{I}$ & $2 \mathrm{I}^{\mathrm{h}} 18^{\mathrm{m}} 49^{\mathrm{s}} .04$ & $+20^{\circ} 0^{\circ} 37^{\prime \prime 6}$ & BB. VI +19.0699 \\
\hline 2 & $212 \mathrm{I} \quad \mathrm{r} 8.3^{\circ}$ & $+20124^{6.0}$ & $W_{2} 2 I^{h} 473$ \\
\hline 3 & $21 \quad 18 \quad 48.73$ & +193421.1 & BB. VI $+19^{\circ} 469^{8}$ \\
\hline 4 & 2 I $16 \quad 43.28$ & +191831.2 & I Pegasi. Berl. Jahrb. \\
\hline 5 & 212248.15 & +181645.9 & Rü. $919^{8}$ \\
\hline 6 & 2 I 38 & $+1034 \times 4.3$ & $W_{1} 2 I^{h} 886$ \\
\hline
\end{tabular}

\begin{tabular}{|c|c|c|c|}
\hline * & $\alpha 1884.0$ & $\delta \mathbf{I} 884.0$ & Autorität \\
\hline 7 & $2 \mathrm{I}^{\mathrm{h}} 3^{6^{\mathrm{m}}} 5^{2^{\mathrm{s}} .98}$ & $+10^{\circ} 17^{\prime} 47^{\prime \prime} 6$ & $W_{1} 2 I^{\mathrm{h}} 859$ \\
\hline 8 & $\begin{array}{lll}21 & 41 & 46.13\end{array}$ & +101321.2 & $W_{1} 25^{\mathrm{h}} 97^{2}$ \\
\hline 9 & $2142 \quad 25.10$ & +10 659.9 & $W_{1} 2 I^{h} 984$ \\
\hline 10 & $2141 \times 3.22$ & +85019.4 & $W_{1} 2 I^{h} 959$ \\
\hline II & $\begin{array}{lll}21 & 55 & 10.53\end{array}$ & +65321.8 & Lal. 42920 \\
\hline 12 & $\begin{array}{lll}2 \mathrm{I} & 52 & \mathrm{3} \\
\end{array}$ & +54524.1 & BB. VI $+5^{\circ} 4909$ \\
\hline
\end{tabular}

Die Beobachtungen sind an einem Ringmikrometer von ro' 8.7 bez. 7' 57 " r Halbmesser gemacht worden, welches auf den festgeklemmten Ocularschieber des Aequatoreals aufgeschranbt wurde. Vergr. ca. 75

Gotha 1884 Dec. 4.

E. Recker. 